\title{
Validierung der deutschen Übersetzung des Childbirth Experience Questionnaire (CEQ2)
}

\author{
Anya Pedersen, Katrin Sieprath und Martina Köhler
}

Lehrstuhl für Klinische Psychologie und Psychotherapie, Christian-Albrechts-Universität zu Kiel

\begin{abstract}
Zusammenfassung: Der Childbirth Experience Questionnaire (CEQ; Dencker, Taft, Bergqvist, Lilja \& Berg, 2010) ist ein reliables und valides Instrument zur Erfassung der Geburtserfahrung. Ziel der Studie war, die revidierte Fassung des CEQ (CEQ2) ins Deutsche zu übersetzen und an einer Stichprobe aus 295 Wöchnerinnen zu validieren. In einer konfirmatorischen Faktorenanalyse konnte die 4-faktorielle Struktur der Originalversion nicht repliziert werden. Stattdessen wurden in einer explorativen Faktorenanalyse vier neue Faktoren gewonnen, die ähnlichen übergeordneten Facetten des Geburtserlebens zugeordnet wurden. Für die neu gewonnenen Skalen erwiesen sich die internen Konsistenzen als überwiegend zufriedenstellend bis gut. Der Zusammenhang zwischen dem CEQ2 und der konstruktnahen Salmon's Item List (Stadlmayr et al., 2001) spricht zudem für die konvergente Validität. Ebenso zeigten sich auch die erwarteten Zusammenhänge mit relevanten Außenkriterien. Die Ergebnisse dieser ersten Validierungsstudie sind somit vielversprechend, wobei weitere Studien in anderen Settings sowie im Längsschnitt wünschenswert sind.
\end{abstract}

Schlüsselwörter: Geburtserfahrung, Geburtserleben, Selbstbeurteilungsfragebogen, Übersetzung

Translation and Validation of the Revised Childbirth Experience Questionnaire (CEQ2)

Abstract: The Childbirth Experience Questionnaire (CEQ; Dencker et al., 2010) is a well-established, validated self-report measure of childbirth experience. The aim of the present study was to translate and validate a German version of the revised CEQ (CEQ2) in a sample of 295 women after delivery during inpatient treatment. The four-factorial structure of the original version was not validated in a confirmatory factor analysis. Instead, four new factors could be identified in an explorative factor analysis, which could be assigned to similar superordinate facets of birth experience. The four new subscales had reasonable internal consistencies. Moreover, significant correlations of the CEQ2 subscales with the Salmon's Item List (Stadlmayr et al., 2001) indicated convergent validity. The CEQ2 was associated with relevant external criteria (e.g., type of birth, oxytocin administration). The results of this first validation study are therefore promising; further studies should be carried out in other settings and with longitudinal designs.

Keywords: birth experience, birth satisfaction, delivery, self-report questionnaire

Im Leben vieler Frauen stellt die Geburt eines Kindes eines der einprägsamsten Ereignisse dar (Simkin, 1992). Vor diesem Hintergrund erscheint es nicht verwunderlich, dass es Hinweise darauf gibt, dass die subjektive Beurteilung der Geburtserfahrung langfristige Auswirkungen auf die Gesundheit und auf das Wohlbefinden von Mutter und Kind haben kann (Simkin, 1991, 1992). Beispielsweise können negative Geburtserfahrungen zu Anpassungsproblemen führen und mit einem erhöhten Risiko für das Auftreten von traumatischen Stress-Symptomen (Creedy, Shochet \& Horsfall, 2000; Czarnocka \& Slade, 2000), postpartalen Depressionen (Righetti-Veltema, Conne-Perréard, Bousquet \& Manzano, 1998) und Posttraumatischen Belastungsstörungen (Ayers \& Pickering, 2001; Pantlen \& Rohde, 2001; Soet, Brack \& Dilorio, 2003; Wijma, Söderquist \& Wijma, 1997) einhergehen.
Auch zukünftige Geburten können aufgrund eines negativen Geburtserlebens durch Ängste negativ beeinflusst werden. So kann es beispielsweise zu einer grundsätzlichen Präferenz der Mutter für eine geplante Kaiserschnittgeburt (primäre Sectio caesarea; Beck, 2004; Goodman, Mackey \& Tavakoli, 2004; Pang, Leung, Lau \& Hang Chung, 2008; Ryding, 1993; Waldenström, Hildingsson \& Ryding, 2006) oder sogar zu einer Vermeidung zukünftiger Schwangerschaften (Gottvall \& Waldenström, 2002) kommen. Dennoch wird die subjektive Geburtserfahrung bisher in Studien selten als outcome-Maß berücksichtigt (Begley et al., 2014).

Larkin, Begley und Devane (2009) definieren das Konstrukt Geburtserfahrung als ein individuelles Lebensereignis, das interagierende subjektive psychologische und physiologische Prozesse umfasst. Die Geburtserfahrung 
wird von einer Vielzahl von Faktoren beeinflusst: Einen wichtigen Einflussfaktor stellt das Gefühl von Kontrolle während der Geburt dar. So beurteilen Frauen, die beispielsweise durch den Erhalt von Informationen und die Einbindung in Entscheidungen während der Geburt ein hohes Ausmaß an Kontrolle erlebt haben, ihre Geburtserfahrung positiver als Frauen, die weniger Kontrolle erlebt haben (Goodman et al., 2004; Green, Coupland \& Kitzinger, 1998; Green \& Baston, 2003; Waldenström, 1999). Des Weiteren sind die wahrgenommene persönliche Unterstützung und hier insbesondere die erlebte Betreuung durch die Entbindungspflegefachkraft von besonderer Relevanz für die individuelle Geburtserfahrung. Dabei geht ein hohes Ausmaß an wahrgenommener Unterstützung mit einer positiveren Geburtserfahrung einher (Brown \& Lumley, 1994; Hodnett, 2002; Hodnett, Gates, Hofmeyr \& Sakala, 2012; Waldenström, 1999; Waldenström, Hildingsson, Rubertsson \& Radestad, 2004). Einen weiteren wichtigen Einflussfaktor stellt die notwendige Rate an geburtsmedizinischen Interventionen dar. Dazu zählen beispielsweise die Gabe von Oxytocin als Wehen auslösendes oder -verstärkendes Hormon und operative Maßnahmen während des Geburtsverlaufs (sekundäre Sectio caesarea oder vaginale operative Entbindungen (Anwendung einer Geburtszange oder einer Saugglocke); Diedrich, 2007). Eine hohe Rate an geburtsmedizinischen Interventionen sowie die Notwendigkeit einer intensivmedizinischen Betreuung des Neugeborenen steigern das Risiko für eine negative Geburtserfahrung (Mori, Tokumasu, Pledge \& Kenyon, 2011; Waldenström et al., 2004; Wiklund, Edman, Ryding \& Andolf, 2008). Darüber hinaus ist eine längere Geburtsdauer eher mit einer negativeren Geburtserfahrung assoziiert als eine kürzere Geburtsdauer (Nystedt, Högberg \& Lundman, 2005; Waldenström, Borg, Olsson, Sköld \& Wall, 1996). Einen weiteren wichtigen Aspekt stellen die persönlichen Erwartungen der Frau an die Geburt dar, wobei realistische Erwartungen mit einer positiveren $\mathrm{Ge}$ burtserfahrung einhergehen (Green et al., 1998; Green \& Baston, 2003; Waldenström, 1999). Hauck, Fenwick, Downie und Butt (2007) konnten in diesem Zusammenhang zeigen, dass Frauen, die schon mindestens ein Kind geboren hatten (Multiparae) und deren Erwartungen sich nach den erlebten Geburten an die Erfahrungen angepasst hatten, ihre Geburtserfahrung häufig positiver beurteilten als Erstgebärende (Primiparae), die ihrerseits häufiger von nicht bestätigten Erwartungen berichteten. Inwiefern die Anzahl der Geburten (Parität) generell einen Einfluss auf die Geburtserfahrung hat, ist noch nicht abschließend geklärt, wobei Waldenström und Kollegen (2004) Erstgebärende als besonders vulnerabel für eine negative Geburtserfahrung einschätzen. Die Befundlage zum Einfluss des Schmerzerlebens während der Geburt auf die Geburtserfahrung ist sehr uneinheitlich. Auf der einen Seite kann eine als schmerzhaft erlebte Geburt als stark belastend empfunden werden (Halperin, Sarid \& Cwikel, 2014; Lavender, Walkinshaw \& Walton, 1999; Slade, MacPherson, Hume \& Maresh, 1993). Dabei deuten Befunde zum Einfluss einer Periduralanästhesie (PDA) darauf hin, dass auch der Erhalt einer PDA zur Schmerzreduktion die Gesamtbeurteilung der Geburtserfahrung trotz der nach der PDA reduzierten Schmerzen nicht zum Positiven verändert (Waldenström et al., 2004). Dies wird u. a. darauf zurückgeführt, dass für die nachträgliche Beurteilung der Geburtserfahrung die Schmerzspitze, d.h. die Stärke der Schmerzen vor Erhalt der PDA, von besonderer Relevanz ist (Waldenström et al., 2004). Auf der anderen Seite gibt es aber ebenso Hinweise darauf, dass eine als schmerzhaft erlebte Geburt auch zu positiven Gefühlen von Erfülltheit und Stolz führen kann (McCrea \& Wright, 1999; Salmon, Miller \& Drew, 1990; Waldenström et al., 1996).

Obwohl international verschiedene ein- und mehrdimensionale Instrumente zur Erfassung der Geburtserfahrung vorliegen (Labour Agentry Scale (LAS), Hodnett \& Simmons-Tropea, 1987; Wijma Delivery Expectancy / Experience Questionnaire (W-DEQ), Wijma et al., 1997), gibt es zum aktuellen Zeitpunkt lediglich ein deutschsprachiges Instrument, welches das intrapartale Geburtserleben mehrdimensional mit seinen unterschiedlichen Facetten erfasst. Bei diesem Instrument handelt es sich um die deutsche Version der Salmon's Item List (Salmon \& Drew, 1992; deutsche Version SIL-GER von Stadlmayr et al., 2001), deren Subskalen in einer Validierungsstudie $(N=251)$ jedoch teilweise geringe interne Konsistenzen aufwiesen, was die Aussagekraft der Skalen infrage stellt. Zudem fokussiert die SIL-GER auf ein sehr enges Zeitfenster nach der Geburt (48 und 96 Stunden postpartum), was sich für einige Untersuchungsfragen als ungünstig erweist.

\section{Der Childbirth Experience Questionnaire}

Dencker, Taft, Bergqvist, Lilja und Berg (2010) haben mit dem Childbirth Experience Questionnaire (CEQ) ein weiteres theoretisch fundiertes Selbstbeurteilungsverfahren in schwedischer Sprache zur Erfassung des Geburtserlebens vorgelegt, dessen Itemkonstruktion anhand von Literaturrecherche und Expertendiskussion erfolgte. Die erste Version wurde 2010 publiziert und an einer Stichprobe von $N=920$ schwedischen Frauen mit einem Beurteilungszeitraum von bis zu einem Monat postpartal validiert. Das Verfahren erfasst mit insgesamt 22 Items die vier Dimensionen Bewältigungsmöglichkeiten (own capacity), Wahrgenommene Sicherheit (perceived safety), Pro- 
fessionelle Unterstützung (professional support) und Partizipation (participation), wobei die Bildung eines Gesamtscores nicht vorgesehen ist. Eine explorative Faktorenanalyse ergab, wie postuliert, eine 4 -faktorielle Struktur mit einer Varianzaufklärung von $54 \%$. Die interne Konsistenz lag mit Ausnahme der Skala Partizipation $(\alpha=.62)$ für alle Skalen bei größer $\alpha=.70$. Die Überprüfung der Kriteriumsvalidität mittels known groups-Analysen zeigte erwartungsgemäß, dass Frauen, die eine längere Geburtsdauer, die Gabe von Oxytocin oder eine operative Entbindung erlebt hatten, ihre Geburtserfahrungen im Mittel als negativer einschätzten (Dencker et al., 2010).

Eine englischsprachige Übersetzung und Validierung erfolgte an einer Stichprobe von $N=350$ Frauen mit einem Retest-Intervall von zwei Wochen (Walker, Wilson, Bugg, Dencker \& Thornton, 2015). In der englischsprachigen Version zeigten alle vier CEQ-Skalen gute bis sehr gute Reliabilitäten (Bewältigungsmöglichkeiten: $\alpha=.79$; Wahrgenommene Sicherheit: $\alpha=.83$; Professionelle Unterstützung: $\alpha=.94$; Partizipation: $\alpha=.72$ ). Die Test-RetestReliabilität für alle Skalen zeigte eine moderate (Partizipation: $\kappa=.60)$ bis substantiale Übereinstimmung (Bewältigungsmöglichkeiten: $\kappa=.66$; Wahrgenommene Sicherheit: $\kappa=.64$; Professionelle Unterstützung: $\kappa=.69$; Gesamtskala: $\kappa=.68$ ). Die Überprüfung der Konstruktvalidität mittels der in der Originalstudie verwendeten known groups-Vergleiche ergab auch in der übersetzten Version, dass Frauen, die eine längere Geburtsdauer, die Gabe von Oxytocin oder eine operative Entbindung erlebt hatten, ihr Geburtserleben auf den Skalen Bewältigungsmöglichkeiten und Wahrgenommene Sicherheit sowie auf der - im Original nicht verwendeten - Gesamtskala im Mittel negativer einschätzten als Frauen ohne diese Erfahrungen. Hinsichtlich der Skala Partizipation zeigte sich dieser Gruppenunterschied nur für operative Entbindungen. Auf der Skala Professionelle Unterstützung ergab die known groups-Analyse keine Gruppenunterschiede, was von den Autoren u.a. darauf zurückgeführt wird, dass in der Schwedischen Validierungsstudie v. a. Frauen nach Spontanpartus ( $<5 \%$ Sectio) befragt wurden, in der englischen Validierungsstudie hingegen $19 \%$ der Frauen nach Sectio befragt wurden. Zudem ergab eine Überprüfung der Kriteriumsvalidität anhand des konstruktnahen Instruments Maternity Survey einen hohen Zusammenhang mit dem CEQ $(r=.73)$.

Um den im Rahmen der beiden Validierungsstudien beobachteten Deckeneffekt auf der Skala Professionelle Unterstützung zu reduzieren und die aus nur drei Items bestehende Skala Partizipation, die mit einem Cronbachs $a$-Wert von .62 eine unzureichende interne Konsistenz aufwies, zu verbessern, wurde eine revidierte Fassung der schwedischen Version angekündigt (Walker et al., 2015). In die revidierte Fassung des CEQ (CEQ2) wurden die
Skalen Bewältigungsmöglichkeiten und Wahrgenommene Sicherheit unverändert übernommen, während die Skala Professionelle Unterstützung um zwei (Item 9, 18) und die Skala Partizipation um ein Item (Item 12) ergänzt wurden. Darüber hinaus wurden fünf Items (Item 10, 11, 13, 14, 17) dieser beiden Skalen umformuliert und drei weitere Items (Item 8, 15, 16) umgepolt. Die Antwortformate wurden beibehalten. In Absprache mit den Autoren der Originalversion wurde in der vorliegen Studie die revidierte englische Fassung des CEQ d.h. der CEQ2, dessen Validierung in Kürze publiziert werden soll, verwendet. Ziel der vorliegenden Studie war es, den CEQ2 in Anlehnung an die von Beaton, Bombardier, Guillemin und Ferraz (2000) vorgeschlagene Prozedur ins Deutsche zu übersetzen und $\mathrm{zu}$ validieren sowie die psychometrischen Eigenschaften des CEQ2 zu überprüfen.

\section{Methode}

\section{Vorgehensweise}

Der CEQ2 wurde entsprechend der Empfehlungen von Beaton und Kollegen (2000) von drei bilingualen Experten unabhängig voneinander ins Deutsche übersetzt und von zwei weiteren bilingualen Experten, denen die Originalversion nicht bekannt war, rückübersetzt (s. ESM 1). Genau wie die englischsprachige Fassung des CEQ2 besteht die deutsche Version aus insgesamt 25 Items, von denen neun Items (Item 3, 5, 8, 9, 15, 16, 21, 22, 23) eine gegenläufige Polung aufweisen und für die Auswertung invertiert werden. Als Antwortformat wurde eine 4-stufige Likert Skala (Stimme gar nicht zu (1) bis Stimme vollkommen zu (4)) und bei drei Items (Schmerzerleben, Gefühl von Kontrolle, Sicherheitsgefühl) eine visuelle Analogskala (VAS; 0-100) verwendet. Die VAS-Scores werden entsprechend der Originalversion wie folgt codiert: 0-40 (1), 41-60 (2), 61-80 (3) und 81-100 (4).

\section{Datenerhebung}

Für die Validierungsstudie wurden Frauen innerhalb der ersten sieben Tage postpartal auf den Wöchnerinnenstationen der Frauenkliniken des UKSH Campus Kiel und des Städtischen Krankenhauses in Kiel rekrutiert. Einschlusskriterien waren ein Mindestalter von 18 Jahren, eine Einlingsschwangerschaft sowie die Geburt eines gesunden und reifen (d.h. Schwangerschaftswoche (SSW) ab $37+0$ bis $41+6$ ) Kindes. Ausschlusskriterien waren sprachliche Barrieren sowie eine primäre Sectio caesarea. Die Teilnahme erfolgte nach ausführlicher Information 
und Aufklärung sowie der schriftlichen Einwilligung zur Teilnahme. Neben der deutschen Version des CEQ2 wurde zur Konstruktvalidierung die deutsche Version der Salmon's Item List (SIL-GER) eingesetzt. Zusätzlich wurden verschiedene soziodemographische Daten (z. B. Alter, Familienstand) erfragt und geburtshilfliche Informationen (d.h. Geburtsmodus (Spontanpartus vs. operative Entbindung; $n=291$ ), Parität (Primi- vs. Multipara; $n=292)$, Geburtsdauer $(n=208)$, Oxytocin-Gabe $(n=290)$, PDA $(n=290))$, soweit verfügbar, aus den Krankenakten entnommen. Das Studienprotokoll wurde vor Beginn der Erhebung von der zuständigen Ethik-Kommission der Medizinischen Fakultät (UKSH-Campus Kiel) geprüft und als ethisch unbedenklich eingeschätzt. Zusätzlich erfolgte eine Prä-Registrierung im Deutschen Register Klinischer Studien (DRKSO0009601).

\section{Stichprobe}

Insgesamt wurden $N=345$ Frauen auf den Wöchnerinnenstationen der beiden regionalen Krankenhäuser über die Studie informiert und zur Teilnahme eingeladen. Sechsundzwanzig Frauen (7\%) lehnten die Teilnahme an der Studie ab, sodass die Fragebögen an 319 Personen verteilt wurden. Der Rücklauf der ausgegebenen Fragebögen lag bei $93 \%$, sodass sich eine Analysestichprobe von $N=295$ Frauen mit einem Durchschnittsalter von $M=30.73$ Jahren $(S D=4.71$; Range: $18-43$ Jahre $)$ ergab. In $77 \%$ der Fälle erfolgte die Geburt als Spontanpartus, d.h. als natürliche vaginale Geburt ohne die Anwendung operativer Maßnahmen (Diedrich, 2007). Für die Mehrzahl der Frauen $(65 \%)$ war es die erste Geburt. Die Geburtsdauer lag bei durchschnittlich $M=7.63$ Stunden (SD $=4.35$; Range: 0-20 Stunden). Oxytocin wurde in $42 \%$ der Geburten gegeben. Eine PDA erfolgte bei $35 \%$ der Frauen.

\section{Weitere Messinstrumente}

Die deutsche Version der Salmon's Item List (SIL-GER; Stadlmayr et al., 2001) ist derzeit in Deutschland das einzige validierte Selbstbeurteilungsverfahren zur Erfassung der Geburtserfahrung. Die Originalversion wurde 1992 von Salmon und Drew entwickelt und von Stadlmayr und Kollegen (2001) ins Deutsche übersetzt. Die SIL-GER umfasst 20 Items, die auf einer sechs-stufigen LikertSkala beurteilt und den zwei intrapartalen Dimensionen Gute emotionale Adaptation und Physisches Unbehagen (Schmerz-Belastungserleben) und den zwei postpartalen Dimensionen Erfülltheit und Negatives emotionales Erleben zugeordnet werden. Die Datenerhebung sollte zwischen
48 und 96 Stunden postpartum erfolgen; die Bearbeitungszeit liegt bei 5 bis 10 Minuten. Die internen Konsistenzen der Subskalen Erfültheit und Gute emotionale Adaptation sind mit Cronbachs's $\alpha$-Werten von $>.80$ als sehr gut einzuschätzen, wohingegen die Subskalen Negatives emotionales Erleben und Physisches Unbehagen weniger zufriedenstellende interne Konsistenzen von $<.70$ aufwiesen (Stichprobe mit $N=251$ Frauen; Stadlmayr et al., 2001). In der vorliegenden Studie lagen alle internen Konsistenzen mit Cronbachs's $\alpha$-Werten $>.75$ im guten bis sehr guten Bereich; lediglich die interne Konsistenz der Subskala Physisches Unbehagen war mit einem Cronbach's $\alpha$-Wert von .48 verbesserungsbedürftig.

\section{Statistische Analysen}

Die statistischen Analysen erfolgten mithilfe des Software-Pakets Statistical Package for the Social Sciences (SPSS Version 22.0). Die Untersuchung der Dimensionalität des Fragebogens (CFA, EFA) wurde mit MPlus (Version 8) durchgeführt. Itemschwierigkeiten wurden mit Microsoft Excel (2016) berechnet. Die Überprüfung der Datenqualität und Itemkennwerte erfolgte auf $\mathrm{Ba}$ sis der deskriptiven Statistiken der Rohwertverteilungen der Einzelitems sowie der Berechnung von Itemschwierigkeiten und -trennschärfen mit part-whole-Korrektur. Zur Untersuchung der Dimensionalität des Fragebogens wurde zunächst die, für die Originalversion berichtete, 4-faktorielle Struktur des CEQ2 (Dencker, persönl. Mitteilung, 26.11.2018) mittels einer konfirmatorischen Faktorenanalyse (CFA) mit robuster Maximum-Likelihood(MLR-)Schätzung überprüft. Zur Skalierung wurden, wie allgemein üblich, Referenzvariablen (Faktor 1: Item 1, Faktor 2: Item 3, Faktor 3: Item 13, Faktor 4: Item 9) genutzt, deren unstandardisierte Ladungen auf 1 fixiert wurden (Bühner, 2011). Entsprechend der Ergebnisse der Validierung des CEQ (Dencker et al., 2010) wurden Korrelationen zwischen den Faktoren zugelassen. Die Überprüfung des Modell-Fit erfolgte auf Basis der gängigen close-fit Indizes (Comparative Fit Index (CFI) $\geq .90$ akzeptable bzw. $\geq .95$ gute Modellpassung, Root Mean Square Error of Approximation (RMSEA) <.10 bzw. nahezu .05, Standardized Root Mean Square Residual (SRMR) $\leq .11$; Bollen, 1989; Hu \& Bentler, 1999). Bei einer nur eingeschränkten Modellpassung wurden Möglichkeiten der Modelloptimierung überprüft (s. ESM 2). Aufgrund der weiterhin nur eingeschränkten Modellpassung wurde anstelle der CFA eine explorative Faktorenanalyse (EFA) mit obliquer Rotation (Geomin-Rotation) durchgeführt. Die Bestimmung der Faktorenanzahl erfolgte auf Basis des Scree-Plots der Eigenwerte der unrotierten Faktoren (Bortz \& Schuster, 2016). Dabei wurden in Anlehnung 
an allgemeine Konventionen Ladungen $\geq .40$ als relevant beurteilt (Fabrigar, Wegener, MacCallum \& Strahan, 1999). Bei relevanten Querladungen wurde für die ItemSkalen-Zuordnung auch die inhaltliche Passung in die Entscheidung mit einbezogen.

Abschließend erfolgte eine Kreuzvalidierung der Faktorstruktur. Dafür wurde die Stichprobe zufällig in eine explorative und eine konfirmatorische Hälfte geteilt. An der ersten Hälfte wurde die oben beschriebene EFA repliziert. An der zweiten Hälfte wurde mithilfe einer CFA die in der EFA gefundene Faktorstruktur überprüft. Aufgrund der durch die Halbierung entstehenden kleinen Substichproben erfolgte die Kreuzvalidierung lediglich im Rahmen zusätzlicher Datenauswertungen. Die Überprüfung der Reliabilität erfolgte über die Bestimmung der internen Konsistenzen. Als Maß für die interne Konsistenz wurden für die einzelnen Subskalen die jeweiligen McDonald's Omega-Werte berechnet. Diese bieten im Vergleich zu Cronbach's Alpha den Vorteil, dass auch bei Interitemkorrelationen das Risiko einer Unter- oder Überschätzung der Reliabilität vergleichsweise gering ist (Dunn, Baguley \& Brunsden, 2014). Die interne Konsistenz wurde bei Reliabilitätskoeffizienten von McDonald's $\omega \geq .70$ als gegeben betrachtet (Watkins, 2017). Zur Vergleichbarkeit mit den vorherigen Validierungsstudien zum CEQ wurden die Cronbach's Alpha-Koeffizienten der einzelnen Subskalen ebenfalls angegeben. Zur Ermittlung der konvergenten Validität wurden die Pearson-Korrelationen zwischen den CEQ2-Subskalen und der SIL-GER berechnet. Dabei wurde die konvergente Validität bei Korrelationen von $r>.50$ als gegeben betrachtet (Bühner, 2011; Moosbrugger \& Kelava, 2012). Die Überprüfung der Kriteriumsvalidität erfolgte über die Berechnung von Pearson-Korrelationen anhand relevanter Variablen aus der Literatur. In Anlehnung an die Validierungsstudien zum CEQ wurden der Geburtsmodus (Spontanpartus vs. operative Entbindung), die Geburtsdauer, sowie die Oxytocin-Gabe als Kriterien verwendet. Zusätzlich dazu wurden die Parität (Primi- vs. Multipara) und der Erhalt einer PDA (nein vs. ja) als Kriterien genutzt. Dabei wurde angenommen, dass Frauen tendenziell eine positivere Geburtserfahrung erleben, wenn sie entweder ihr Kind spontan gebären, die Geburt weniger lange dauert oder sie kein Oxytocin und keine PDA unter der Geburt erhalten haben (Dencker et al., 2010; Waldenström et al., 2004; Walker et al., 2015). Weiter wurde angenommen, dass Erstgebärende vulnerabler für eine negative $\mathrm{Ge}$ burtserfahrung sind (Waldenström et al., 2004). Über die Berechnung der Pearson-Korrelationen hinaus wurde für die Kriteriumsvariablen mittels verschiedener Regressionsmodelle die inkrementelle Validität der CEQ2-Skalen im Vergleich zur SIL-GER bestimmt. Dabei sollten die CEQ2-Skalen zusätzlich zur SIL-GER einen signifikanten
Beitrag zur Vorhersage der einzelnen Kriterien leisten. Als Signifikanzniveau für alle Analysen wurde ein $\alpha$-Niveau von $p<.05$ festgelegt.

\section{Ergebnisse}

\section{Datenqualität und Itemkennwerte}

Im Rahmen der Datenaufbereitung wurde zunächst der gesamte Datensatz auf fehlende Werte hin analysiert und einzelne fehlende Werte, im Sinne eines Auslassens einzelner Fragebogenitems in den Fragebögen (<50\%), wurden durch multiple Imputation mithilfe des MarkovChain-Monte-Carlo (MCMC)-Algorithmus ersetzt (Anzahl der imputierten Werte: CEQ2: $n=24$; SIL-GER: $n=25$; Schafer \& Graham, 2002). Hierbei wurden jeweils fünf Imputationsschritte je Fragebogen durchgeführt, deren letzte Werte als Realisation für die fehlenden Beobachtungen verwendet wurden. Auf diese Weise konnten für den CEQ2 $100 \%$ vollständige Datensätze und für die SIL-GER $96 \%(n=282)$ erreicht werden. Die SIL-GER wurde von 13 Probandinnen nicht bearbeitet. Die Itemschwierigkeiten lagen im mittleren bis hohen Bereich (44.9-94.8), was auf eine teilweise eingeschränkte Differenzierungsfähigkeit (Item 9, 12, 13, 14, 15, 16, 17, 18, 19, 22) hinweist. Die eingeschränkte Differenzierungsfähigkeit zeigte sich vor allem bei den Items der Subskala Professionelle Unterstützung, was damit in Zusammenhang stehen könnte, dass alle befragten Wöchnerinnen professionelle Unterstützung durch das Krankenhauspersonal in Anspruch genommen und als Ergebnis ein gesundes Kind geboren hatten. Da Deckeneffekte somit möglicherweise nicht nur ungünstige Itemformulierungen, sondern auch Besonderheiten der untersuchten Stichprobe reflektieren könnten, wurde von einer Itemselektion auf Basis der Itemschwierigkeiten abgesehen. Die Trennschärfen lagen für alle Items im zufriedenstellenden Bereich $>.30$ (Döring \& Bortz, 2016). Eine ausführliche Darstellung der deskriptiven Kennwerte sowie unstandardisierten Faktorladungen der explorativen Faktorenanalyse der Einzelitems des CEQ2 sind in ESM 3 zu finden.

\section{Dimensionalität und Skalenkennwerte}

Im CFA-Modell wurde jedes latente Konstrukt durch seine entsprechenden manifesten Items des CEQ2 definiert (s. ESM 2). Der CFI-Wert lag mit einem Wert von .801 im nicht akzeptablen Bereich < .90 (Bollen, 1989; Hu \& Bentler, 1999). Im Gegensatz dazu sprachen der RMSEA mit einem Wert von .080 (<.10) sowie der SRMR 
von .082 (<.11) für eine adäquate Modellgüte (Bollen, 1989; Hu \& Bentler, 1999). Somit konnte die für den CEQ2 postulierte 4-faktoriellen Struktur (Dencker, persönl. Mitteilung, 26.11.2018) nur eingeschränkt bestätigt werden. Da sich zudem hohe Interkorrelationen von bis zu $r=.97$ zwischen den einzelnen Faktoren zeigten (s. ESM 2), wurden weitere Optionen einer Modelloptimierung geprüft (u.a. das Zulassen von Korrelationen zwischen den Fehlervarianzen der negativ formulierten Items, die Bildung von Faktoren 2. Ordnung, die Optimierung der Item-Skala-Zuordnung vor dem Hintergrund der Modifikationsindizes, die Selektion von Einzelitems auf Basis von geringen Faktorladungen $[<.40 ;$ d.h. Item 10], sowie abweichenden Item-Formulierungen [Item 23-25]). Trotz der Modelloptimierungen konnte keine relevante Verbesserung des Modell-Fit erreicht werden, sodass die vorgeschlagene 4-faktorielle Struktur nur unzureichend bestätigt werden konnte.

Aus diesem Grund wurde eine explorative Faktorenanalyse (EFA) durchgeführt, um die latente Struktur der deutschen Version des CEQ2 zu ermitteln. Hierzu wurden zunächst die als Globalmaß formulierten Items (Item 2325) aus der Skala eliminiert, da sie sich nicht nur in der Art der Formulierung, sondern auch im Hinblick auf das Antwortformat von den restlichen 22 Items substantiell unterschieden. Mit den verbliebenen 22 Items wurde eine explorative Faktorenanalyse mit obliquer Rotation (Geomin-Rotation) durchgeführt. Dabei zeigte sich eine sehr gute statistische, sowie inhaltliche Passung eines 4-faktoriellen Modells mit leicht geänderter Zuordnung der Items zu den vier Faktoren (s. ESM 3). Die Benennung der Faktoren konnte überwiegend beibehalten werden; lediglich der Faktor Wahrgenommene Sicherheit wurde, da er überwiegend Items zum emotionalen Erleben während der Geburt enthielt (z.B. Item 6: „Ich fühlte mich während der Wehen und der Entbindung glücklich"), entsprechend in Emotionales Erleben umbenannt. Vier Items (Item 1, 5, 8, 22) wiesen in diesem Modell Faktorladungen $<.40$ auf, sodass sie zur Skalenberechnung ausgeschlossen wurden. Ggf. kann erwogen werden, die in der deutschen Version ausgeschlossenen vier Items in internationalen Studien dennoch mitzuführen, um ergänzend Vergleiche hinsichtlich der Beantwortung einzelner Items zu ermöglichen. Die gefundene Faktorstruktur konnte in der zur Kreuzvalidierung halbierten Stichprobe explorativ repliziert $(N=148)$ und anschließend konfirmatorisch $(N=147)$ bestätigt werden $(\mathrm{CFI}=.90$; RMSEA $=.07$; SRMR = .07).

Die Berechnung der Skalenwerte erfolgte, wie in den Ursprungsversionen des Fragebogens, durch skalenweise Mittelwertbildung. Die Skalenausnutzungen können als gegeben und die entsprechenden Verteilungen als symmetrisch betrachtet werden (vgl. Tabelle 1). Lediglich die
Skala Professionelle Unterstützung wies mit einer Schiefe von -1.82 sowie einer Kurtosis von 3.78 eine linksschiefe Verteilung auf, was, wie oben dargestellt, möglicherweise durch Stichprobencharakteristika bedingt sein könnte. Die Interskalenkorrelationen (vgl. Tabelle 1) sind moderat bis hoch (Cohen, 2013), aber insgesamt deutlich niedriger als die Korrelationen zwischen den in der Originalversion des CEQ2 postulierten Subskalen (s. ESM 2).

\section{Reliabilität und Validität}

Abgesehen von der Skala Partizipation, die einen McDonald's $\omega$-Koeffizienten von .67 (95\%-VI = .58-.85; Cronbach's $\alpha=.67$ ) aufwies, erwiesen sich alle anderen Skalen mit Reliabilitätskennwerten von McDonald's $\omega>.70$ als reliabel (vgl. Tabelle 1). Hinsichtlich der konvergenten Validität zeigten sich erwartungsgemäß moderate bis hohe negative Korrelationen der Skalenwerte des CEQ2 mit dem SIL-GER-Gesamtwert (vgl. Tabelle 1). Die Überprüfung der Kriteriumsvalidität erfolgte über die Berechnung Pearson-Korrelationen, wobei sich die erwarteten $\mathrm{Zu}-$ sammenhänge nur zum Teil zeigten: Nach einem Spontanpartus wurde das Geburtserleben auf den Skalen Bewältigungsmöglichkeiten, Partizipation und Emotionales Erleben positiver eingeschätzt als nach einer operativen Entbindung. Erwartungsgemäß wurde das Geburtserleben auch von denjenigen Frauen, die während der Entbindung kein Oxytocin und keine PDA erhalten hatten oder eine vorangegangene Geburt erlebt hatten (Multiparae), auf der Skala Emotionales Erleben positiver eingeschätzt (vgl. Tabelle 1). Entgegen der Erwartungen konnten für die Geburtsdauer keine Zusammenhänge mit den CEQ2-Skalenwerten gezeigt werden. Zwischen den geburtsmedizinischen Variablen (Geburtsmodus, Oxytocingabe, PDA) fanden sich kleine bis mittlere positive Korrelationen zwischen .13 und .50. Hinsichtlich der Parität zeigten sich erwartungsgemäß kleine bis mittlere negative Zusammenhänge zwischen -.17 und - .38 mit den anderen geburtsmedizinischen Variablen.

Abschließend wurde die inkrementelle Validität durch Regressionsanalysen überprüft. Zur Vorhersage der binären Außenkriterien (Geburtsmodus, Oxytocin-Gabe, Parität, PDA) wurden logistische Regressionsanalysen berechnet. Zur Vorhersage der Geburtsdauer wurde eine lineare Regressionsanalyse durchgeführt. In den Analysen wurden zunächst jeweils der SIL-GER-Gesamtwert (Modell 1) und in einem zweiten Schritt zusätzlich die vier Skalen des CEQ2 (Modell 2) als Prädiktoren für die verschiedenen Kriterien berücksichtigt. Während Modell 1 lediglich für den Geburtsmodus signifikant wurde (Modell- $\chi^{2}=4.44, d f=1, p=.04$; Nagelkerkes $R^{2}=.02$ ), führte 
Tabelle 1. Deskriptive Skalenkennwerte und Korrelationen der CEQ2-Skalen mit dem SIL-GER sowie mit geburtsmedizinischen Variablen

\begin{tabular}{|c|c|c|c|c|c|c|c|c|c|c|c|c|}
\hline & Skala & $N$ & M & $S D$ & Schiefe & Kurtosis & $\begin{array}{c}\text { McDonald's } \omega \\
{[95 \%-\mathrm{VI}]}\end{array}$ & $\alpha$ & 1 & 2 & 3 & 4 \\
\hline 1. & Bewältigungsmöglichkeiten & 295 & 2.70 & .65 & -.40 & -.11 & $\begin{array}{c}.77 \\
{[.72-.82]}\end{array}$ & .77 & 1.00 & & & \\
\hline 2. & Professionelle Unterstützung & 295 & 3.66 & .41 & -1.82 & 3.78 & $\begin{array}{c}.83 \\
{[.78-.87]}\end{array}$ & .83 & $.32 * \star$ & 1.00 & & \\
\hline 3. & Partizipation & 295 & 3.25 & .72 & -.90 & .20 & $\begin{array}{c}.67 \\
{[.58-.75]}\end{array}$ & .67 & $.27 \star \star$ & $.43 * \star$ & 1.00 & \\
\hline 4. & Emotionales Erleben & 295 & 2.50 & .72 & -.17 & -.57 & $\begin{array}{c}.82 \\
{[.78-.85]}\end{array}$ & .79 & $.55^{\star \star}$ & $.31 \star \star$ & $.39 * \star$ & 1.00 \\
\hline 5. & SIL-GER-Gesamtwert & 282 & 43.93 & 19.21 & .58 & .03 & & .89 & $-.46^{\star \star}$ & $-.34^{\star \star}$ & $-.36^{\star \star}$ & $-.58 * \star$ \\
\hline 6. & Geburtsmodus ${ }^{1}$ & 291 & .23 & .42 & - & - & - & - & $-.13 *$ & -.10 & $-.31 * \star$ & $-.23 * *$ \\
\hline 7. & Geburtsdauer & 208 & 7.09 & 4.41 & - & - & - & - & -.01 & .02 & -.01 & -.09 \\
\hline 8. & Oxytocingabe $^{1}$ & 290 & .42 & .50 & - & - & - & - & -.04 & -.02 & -.10 & $-.13^{\star}$ \\
\hline 9. & Parität $^{1}$ & 292 & .36 & .48 & - & - & - & - & .04 & -.11 & .09 & $.12^{\star}$ \\
\hline 10. & $\mathrm{PDA}^{1}$ & 290 & .35 & .48 & - & - & - & - & -.10 & -.06 & -.05 & $-.17 * *$ \\
\hline
\end{tabular}

Anmerkungen: CEQ2 = revidierte Version des Childbirth Experience Questionnaire (Deutsche Version), SIL-GER = Salmon's Item List - Deutsche Version, PDA = Periduralanästhesie; $N=$ Stichprobengröße, $M=$ Mittelwert, $S D=$ Standardabweichung, $95 \%$-VI = Bootstrap-95\%-Vertrauensintervall; $\alpha=$ Cronbach's $\alpha .{ }^{1}$ Punktbiseriale Korrelation: Geburtsmodus: Spontanpartus (0) vs. operative Entbindung (1); Oxytocingabe: nein (0) vs. ja (1); Parität: Primipara (0) vs. Multipara (1); PDA: nein (0) vs. ja (1); ${ }^{\star} p<.05,{ }^{\star *} p<.01$ (Pearson-Korrelationskoeffizient, 2-seitig).

die Hinzunahme der CEQ2-Skalen als Prädiktorvariablen in zwei von fünf Modellen zu einem relevanten Anstieg in der Varianzaufklärung (Geburtsmodus: Modell$\chi^{2}=23.00, d f=4, p<.001$; Nagelkerkes $R^{2}=.14$; Geburtsdauer: $F(5,193)=.57, p=.73 ; R^{2}=.01$; OxytocinGabe: Modell- $\chi^{2}=7.67, d f=4, p=.10$; Nagelkerkes $R^{2}=.04$; Parität: Modell- $\chi^{2}=22.82, d f=4, p<.001 ; \mathrm{Na}-$ gelkerkes $R^{2}=.11$; PDA: Modell- $\chi^{2}=6.93, d f=4, p=.14$; Nagelkerkes $\left.R^{2}=.04\right)$.

\section{Diskussion}

Im Leben vieler Frauen stellt die Geburt eines Kindes eines der einprägsamsten Lebensereignisse dar. Eine negative Geburtserfahrung kann ein Risikofaktor für das Wohlbefinden von Mutter und Kind sein und zukünftige Schwangerschaften und Geburten negativ beeinflussen. Vor diesem Hintergrund ist es wichtig, Instrumente zur Verfügung zu haben, die die Geburtserfahrung valide erfassen, um Mütter mit einer besonders negativen Geburtserfahrung frühzeitig identifizieren und ihnen entsprechende Interventionen anbieten zu können. Übersetzte und validierte Instrumente zur Erfassung der Geburtserfahrung liegen in Deutschland allerdings kaum vor. Daher war das Ziel dieser Arbeit, den CEQ2, der eine Revision des international vielfach eingesetzten CEQ darstellt, aber bislang nur in englischer Sprache vorliegt, ins Deutsche zu übersetzen, zu validieren und insbesondere die 4-faktorielle Skalenstruktur zu überprüfen.

Bezüglich der psychometrischen Eigenschaften des CEQ2 zeigten sich im Einzelnen folgende Ergebnisse:

Die Analyse der Datenqualität und Itemkennwerte erbrachte für den Großteil der Items zufriedenstellende Ergebnisse, wobei diejenigen Items, die sich auf den Erhalt professioneller Unterstützung bezogen, größtenteils Deckeneffekte (Itemschwierigkeiten $>$.80) und damit eine eingeschränkte Differenzierungsfähigkeit aufwiesen. Derartige Deckeneffekte zeigten sich schon in den unterschiedlichen Validierungsstudien des CEQ (Dencker et al., 2010; Walker et al., 2015), d.h., dass durch die Revision des Fragebogens - zumindest in der Deutschen Version - nicht die gewünschte Reduktion der Deckeneffekte erreicht werden konnte. Walker und Kollegen (2015) haben in Bezug auf die Deckeneffekte im CEQ2 argumentiert, dass diese Ausdruck einer insgesamt hohe Zufriedenheit der Studienteilnehmerinnen mit der erhaltenen professionellen Unterstützung sein könnten. Da diese hohe Zufriedenheit mit der professionellen Unterstützung nicht unbedingt für jede Untersuchungssituation angenommen werden kann, wurden die entsprechenden Items, die möglicherweise in Situationen mit geringerer professioneller Unterstützung relevant sein könnten, im Fragebogen belassen. Die Beibehaltung dieser Items erhöht zudem die internationale Vergleichbarkeit.

Im Hinblick auf die Skalenstruktur konnte die für den CEQ2 postulierte 4-faktorielle Struktur des Fragebogens (Dencker, persönl. Mitteilung, 26.11.2018) auch unter der 
Anwendung verschiedener Möglichkeiten der Modelloptimierung nicht repliziert werden. Stattdessen konnten in einer explorativen Faktorenanalyse vier neue Faktoren gewonnen werden, die inhaltlich zwar, abgesehen von der Umbenennung des Faktors Wahrgenommene Sicherheit in Emotionales Erleben, ähnlichen übergeordneten Facetten des Geburtserlebens (d.h. Bewältigungsmöglichkeiten, Professionelle Unterstützung, Partizipation) zugeordnet werden konnten, aber hinsichtlich der Zuordnung der Items $\mathrm{zu}$ den Skalen, an einigen Stellen, Unterschiede aufwiesen. In diesem Zusammenhang ist nicht auszuschließen, dass die unterschiedlichen faktoriellen Strukturen kulturabhängige Unterschiede in der Bewertung der Geburtserfahrung abbilden. So wäre es beispielsweise denkbar, dass in den verschiedenen Kulturen ein unterschiedliches konzeptionelles Verständnis der Geburtserfahrung vorliegt, das auch mit den sehr unterschiedlichen medizinischen Versorgungsstrukturen zusammenhängen könnte (Finkenstädt, 2017). Zur weiteren Klärung potentieller kultureller Besonderheiten sollte in zukünftigen Studien eine empirische Überprüfung der Messinvarianz anhand der Fragebogendaten von Stichproben aus unterschiedlichen Kulturkreisen erfolgen.

Für die neu gewonnenen Skalen erwiesen sich die internen Konsistenzen, in Anlehnung an allgemeine Konventionen (Moosbrugger \& Kelava, 2012), für alle Skalen als überwiegend zufriedenstellend bis gut. Während die Skalen Bewältigungsmöglichkeiten, Emotionales Erleben (im CEQ: Wahrgenommene Sicherheit) und Professionelle Unterstützung auch in der Originalversion des CEQ vergleichbare interne Konsistenzen zeigten, konnte durch die Revision des CEQ und die neue Item-Skala-Zuordnung für die Skala Partizipation eine geringfügige Verbesserung der Reliabilität erreicht werden (Cronbach's $\alpha=.62$; Dencker et al., 2010; in der aktuellen Studie Cronbach's $\alpha=.67$ ).

Im Hinblick auf die konvergente Validität zeigten sich erwartungskonforme negative Zusammenhänge zwischen der Beurteilung der Geburtserfahrung auf den verschiedenen Skalen des CEQ2 und der Beurteilung des Geburtserlebens in der SIL-GER. Die Zusammenhänge erreichten auf den Skalen Bewältigungsmöglichkeiten und Emotionales Erleben Werte nahe .50, was als Bestätigung der konvergenten Validität angesehen werden kann (Bühner, 2011; Moosbrugger \& Kelava, 2012). Für die Skalen Professionelle Unterstützung und Partizipation ist der Zusammenhang etwas geringer ausgeprägt, was inhaltlich erklärbar scheint, da die Items der SIL-GER primär emotionale $\mathrm{Zu}$ stände des Geburtserlebens erfassen.

Bezüglich der Kriteriumsvalidität beurteilten Frauen, die ihr Kind spontan zur Welt gebracht hatten, erwartungsgemäß ihre Geburtserfahrung auf den Skalen Bewältigungsmöglichkeiten, Emotionales Erleben und Partizipation positiver als Frauen, die ihr Kind durch eine un- geplante operative Entbindung (d.h. sekundäre Sectio caesarea oder vaginale operative Entbindungen) zur Welt gebracht hatten. Lediglich bzgl. der Skala Professionelle Unterstützung zeigte sich kein Gruppenunterschied, was darauf hindeuten könnte, dass die Unterstützung durch medizinisches Fachpersonal bei einer operativen Entbindung als vergleichbar mit einer spontanen Geburt wahrgenommen wurde. Bezüglich der Oxytocin-Gabe, dem Erhalt einer PDA unter der Geburt und der Parität entsprachen die Befunde den Erwartungen: Frauen, die während der Geburt kein Oxytocin und keine PDA erhalten hatten sowie Frauen, die schon mal ein Kind geboren hatten, schätzten ihre Geburtserfahrung auf der Skala Emotionales Erleben positiver ein als Frauen, die während der Geburt Oxytocin erhalten hatten. Während Dencker und Kollegen (2010) im Rahmen der Validierung der schwedisch-sprachigen Originalversion des CEQ Hinweise darauf fanden, dass Frauen, die ihr Kind spontan und ohne zusätzliche Gabe von Oxytocin entbunden haben, signifikant höhere Werte auf allen Skalen des CEQ aufwiesen, konnten wir im Einklang mit den Befunden aus der Validierung der englischsprachigen CEQ-Version (Walker et al., 2015) die Befunde nur teilweise replizieren. Allerdings erwies sich das Geburtserleben, entgegen der Befunde aus den Validierungsstudien zum CEQ (Dencker et al., 2010; Walker et al., 2015), in unserer Stichprobe als unabhängig von der Geburtsdauer.

Walker und Kollegen (2015) nannten als mögliche Gründe für die beobachteten Unterschiede zu den Ergebnissen von Dencker und Kollegen (2010) Unterschiede in den Stichprobencharakteristika, die auch für die vorliegende Studie relevant sein könnten. In die Originalstudie (Dencker et al., 2010) wurden nur Erstgebärende bei unkomplizierter Schwangerschaft (low risk) mit spontanem Geburtsbeginn eingeschlossen. Bei der Untersuchung von Walker und Kollegen (2015) wurden auch Frauen eingeschlossen, deren Schwangerschaft nicht zwingend als unkompliziert einzuordnen war. In der vorliegenden Untersuchung wurden darüber hinaus neben Erstgebärenden auch Mehrfachgebärende sowie Frauen, deren Geburtsbeginn eingeleitet worden war oder durch Blasensprung begann, eingeschlossen. Weitere mögliche Ursachen für die Unterschiede in den Gruppenvergleichen könnten im postpartalen Zeitpunkt der Erhebung oder in der Datenerhebungsmethode liegen. Neben diesen Unterschieden in der Stichprobenzusammensetzung erfolgte die Datenerhebung in der vorliegenden Studie innerhalb der ersten sieben Tage nach der Geburt im stationären Kontext, wohingegen Dencker und Kollegen (2010) die Teilnehmerinnen einen Monat postpartal per Email oder postalisch kontaktierten. Dieser Rekrutierungsunterschied zeigt sich auch in der deutlich höheren Rücklaufquote der vorliegenden Studie (93\% vs. $78 \%$ ), wodurch das Risiko 
einer systematischen Stichprobenselektion reduziert werden konnte. Insgesamt wird vor dem Hintergrund der oben dargestellten Befunde die Validität des CEQ2 als gegeben angesehen, wobei insbesondere auch die weiterführenden Analysen zur inkrementellen Validität für einen diagnostischen Zugewinn im Hinblick auf die Erfassung des Geburtserlebens sprechen.

\section{Stärken, Limitationen und Ausblick}

Im Rahmen der vorliegenden Untersuchung wurde ein standardisierter Übersetzungsprozess (Beaton et al., 2000) eingesetzt, um Einschränkungen der Reliabilität und Validität durch mögliche Übersetzungsfehler zu minimieren. Auch konnten Unstimmigkeiten, die im Rahmen der Übersetzung der schwedischen Version ins Englische entstanden waren, in Rücksprache mit der Autorin der Originalversion für die Deutsche Version korrigiert werden. Positiv ist zudem, dass es gelungen ist, eine große Stichprobe konsekutiv zu erheben, die geringe Selektionseffekte beinhaltet (sehr hohe Rücklaufquote), wobei die Datenerhebung in zwei unterschiedlichen Krankenhäusern die Repräsentativität der Stichprobe erhöht. Zudem zeigte sich eine hohe Akzeptanz der Teilnehmerinnen bzgl. des CEQ2 zur Studienteilnahme.

Dennoch gibt es einige Limitationen, die berücksichtigt werden sollten: Für die deutsche Übersetzung des CEQ2 fand sich eine andere Faktorstruktur als in der englischen Version des CEQ2. Zwar konnten auch vier Faktoren extrahiert werden, diese unterschieden sich allerdings bezüglich der Itemzuordnung von den in der Originalversion des Fragebogens gefundenen vier Faktoren. Die Überprüfung erfolgte mittels einer Kreuzvalidierung mit Split-Half-Methode, wobei sich durch die Halbierung der Stichprobe relativ kleine Teilstichprobengrößen ergaben. In Folgestudien wäre eine Überprüfung der gefundenen Faktorstruktur in ausreichend großen Samples wünschenswert. Des Weiteren konnte die auch schon in den Validierungsstudien der schwedischen und englischen Fragebogenversionen beobachtete geringe interne Konsistenz der Skala Partizipation nur geringfügig verbessert werden. Vor dem Hintergrund der fortbestehenden Kürze der Skala wäre es in Folgestudien zu erwägen, der Skala ggf. weitere Items hinzuzufügen. Darüber hinaus konnte auch der Deckeneffekt auf der Skala Professionelle Unterstützung nicht reduziert werden. Allerdings ist nicht auszuschließen, dass die hohen Werte, insbesondere auf der Skala Professionelle Unterstützung durch den Zeitpunkt der Datenerhebung (stationären Kontext kurz nach der Geburt) beeinflusst wurden. Daher wurden die Items dieser Skala trotz der eher geringen Differenzierungsfähigkeit bei Frauen kurz nach der Entbindung im Fragebogen be- lassen, nicht zuletzt um die Vergleichbarkeit mit der Originalversion $\mathrm{zu}$ erhalten. Schlussendlich konnte Simkin (1992) zeigen, dass sich die Beurteilung der Geburtserfahrung über die Zeit verändert. Einige Aspekte der Geburtserfahrung wurden einige Jahre nach der Geburt negativer bewertet als kurz nach der Geburt, wobei die befragten Frauen sich auch Jahre später genau an die Geburt erinnern konnten. Da die Sorge um die Gesundheit des Kindes eine der größten Sorgen von Schwangeren kurz vor der Geburt darstellt (Maimburg, Væth, Hvidman, Dürr \& Olsen, 2013; Öhman, Grunewald \& Waldenström, 2003; Peñacoba-Puente, Monge \& Morales, 2011), scheint kurz nach der Geburt die Freude und Erleichterung über die Geburt eines gesunden Kindes im Vordergrund zu stehen (Waldenström, 1999). Darüber hinaus gibt es Hinweise darauf, dass die Unterstützung des betreuenden Personals bei Datenerhebungen im stationären Kontext nicht offen beurteilt wird (Robinson, Salmon \& Yentis, 1998). Beide Aspekte können daher zu einer zunächst positiveren Beurteilung der Geburtserfahrung beitragen (Green, 2012; Hodnett, 2002; Waldenström et al., 2004). Vor diesem Hintergrund erscheinen längsschnittliche Untersuchungen des Geburtserlebens sinnvoll und wünschenswert.

\section{Fazit für die Praxis}

Die Erfassung des Geburtserlebens ist insbesondere aufgrund des in Deutschland bestehenden Mangels an verfügbaren validierten Instrumenten von hoher klinischer Relevanz, um die Betreuungsqualität sowohl während als auch nach der Geburt weiter zu verbessern. Der CEQ2 ist ein praktikables Selbstbeurteilungsverfahren, das mit einer Bearbeitungszeit von ca. 10 Minuten eine ökonomisch und von den Befragten gut akzeptierte Erfassung der Geburtserfahrung ermöglicht. Die mehrdimensionale Erhebung mit dem CEQ2 bietet zudem, beispielsweise im Rahmen von Evaluationsstudien und Verlaufsuntersuchungen, den Vorteil, dass eine detaillierte Erfassung verschiedener Aspekte des Geburtserlebens möglich ist. Die Ergebnisse dieser ersten Validierungsstudie sind vielversprechend, wobei die gefundene Faktorenstruktur in weiteren Studien repliziert werden sollte. Darüber hinaus wären Untersuchungen im Längsschnitt sowie ggf. auch Untersuchungen, bei denen das Ausmaß der professionellen Unterstützung zwischen Einrichtungen variiert, wünschenswert. Insgesamt unterstreichen die Ergebnisse der vorliegenden Studie die gute Eignung des CEQ2 für die klinische Praxis. 


\section{Elektronische Supplemente}

Die elektronischen Supplemente sind mit der OnlineVersion dieses Artikels verfügbar unter https://doi.org/ 10.1026/0012-1924/a000267

ESM 1. Vorgehen bei der Übersetzung des CEQ2 nach Beaton et al. (2000).

ESM 2. Konfirmatorisches Faktorenmodell gemäß der Originalvalidierung des $\mathrm{CEQ} 2$ inklusive der standardisierten Faktorladungen sowie der Faktoreninterkorrelationen. ESM 3. Deskriptive Kennwerte sowie unstandardisierte Faktorladungen der explorativen Faktorenanalyse der Einzelitems des CEQ2.

ESM 4. Deskriptive Skalenkennwerte und Interkorrelationen der zur Validierung des CEQ2 betrachteten geburtsmedizinischen Variablen.

\section{Literatur}

Ayers, S. \& Pickering, A. D. (2001). Do women get posttraumatic stress disorder as a result of childbirth? A prospective study of incidence. Birth, 28(2), 111-118. https://doi.org/10.1046/j. 1523-536X.2001.00111.x

Beaton, D. E., Bombardier, C., Guillemin, F. \& Ferraz, M. B. (2000). Guidelines for the process of cross-cultural adaptation of selfreport measures. Spine, 25, 3186-3191. https://doi.org/10. 1097/00007632-200012150-00014

Beck, C. T. (2004). Birth trauma: In the eye of the beholder. Nursing research, 53(1), 28 -35. https://doi.org/10.1097/00006199-200 401000-00005

Begley, C. M., Gross, M. M., Dencker, A., Benstoem, C., Berg, M. \& Devane, D. (2014). Outcome measures in studies on the use of oxytocin for the treatment of delay in labour: A systematic review. Midwifery, 30, 975-982. https://doi.org/10.1016/j.midw. 2014.06.005

Bollen, K. A. (1989). Structural equations with latent variables. New York, NY: Wiley.

Bortz, J. \& Schuster, C. (2016). Statistik für Human- und Sozialwissenschaftler: Extras online (Lehrbuch, 7., vollst. überarb. und erw. Aufl.). Berlin: Springer.

Brown, S. \& Lumley, J. (1994). Satisfaction with care in labor and birth: A survey of 790 Australian women. Birth, 21(1), 4-13. https://doi.org/10.1111/j.1523-536X.1994.tb00909.x

Bühner, M. (2011). Einführung in die Test- und Fragebogenkonstruktion (PS Psychologie, 3., aktual. und erw. Aufl.). München: Pearson Studium.

Cohen, J. (2013). Statistical power analysis for the behavioral sciences (2nd ed.). Hoboken, NJ: Taylor and Francis.

Creedy, D. K., Shochet, I. M. \& Horsfall, J. (2000). Childbirth and the development of acute trauma symptoms: Incidence and contributing factors. Birth, 27(2), 104-111. https://doi.org/10. 1046/j.1523-536x.2000.00104.x

Czarnocka, J. \& Slade, P. (2000). Prevalence and predictors of post-traumatic stress symptoms following childbirth. British Journal of Clinical Psychology, 39(1), 35 -51. https://doi.org/10. 1348/014466500163095
Dencker, A., Taft, C., Bergqvist, L., Lilja, H. \& Berg, M. (2010). Childbirth Experience Questionnaire (CEQ): Development and evaluation of a multidimensional instrument. BMC pregnancy and childbirth, 10(681), 1-8. https://doi.org/10.1186/14712393-10-81

Diedrich, K. (Hrsg.). (2007). Gynäkologie und Geburtshilfe: Mit 97 Tabellen (Springer E-book Collection, 2., vollst. neu bearb. Aufl.). Berlin: Springer.

Döring, N. \& Bortz, J. (2016). Forschungsmethoden und Evaluation in den Sozial- und Humanwissenschaften (Springer-Lehrbuch, 5. vollst. überarb., aktual. und erw. Aufl.). Berlin: Springer. https://doi.org/10.1007/978-3-642-41089-5

Dunn, T. J., Baguley, T. \& Brunsden, V. (2014). From alpha to omega: A practical solution to the pervasive problem of internal consistency estimation. British journal of psychology, 105, 399 412. https://doi.org/10.1111/bjop.12046

Fabrigar, L. R., Wegener, D. T., MacCallum, R. C. \& Strahan, E. J. (1999). Evaluating the use of exploratory factor analysis in psychological research. Psychological Methods, 4, 272-299. https://doi.org/10.1037/1082-989X.4.3.272

Finkenstädt, V. (2017). Zugangshürden in der Gesundheitsversorgung: Ein europäischer Überblick. Köln: Wissenschaftliches Institut der PKV (WIP).

Goodman, P., Mackey, M. C. \& Tavakoli, A. S. (2004). Factors related to childbirth satisfaction. Journal of advanced nursing, 46, 212 - 219. https://doi.org/10.1111/j.1365-2648.2003.02981.x

Gottvall, K. \& Waldenström, U. (2002). Does a traumatic birth experience have an impact on future reproduction? BJOG: An International Journal of Obstetrics and Gynaecology, 109, $254-$ 260. https://doi.org/10.1111/j.1471-0528.2002.01200.x

Green, J. M. (2012). Integrating women's views into maternity care research and practice. Birth, 39, 291 -295. https://doi.org/10. 1111/birt.12003

Green, J. M. \& Baston, H. A. (2003). Feeling in control during labor: Concepts, correlates, and consequences. Birth, 30, 235-247. https://doi.org/10.1046/j.1523-536X.2003.00253.x

Green, J. M., Coupland, V. A. \& Kitzinger, J. (1998). Great expectations: A prospective study of women's expectations and experiences of childbirth (2nd ed.). Hale, Cheshire: Books for Midwives.

Halperin, O., Sarid, O. \& Cwikel, J. (2014). A comparison of Israeli Jewish and Arab women's birth perceptions. Midwifery, 30, 853 - 861. https://doi.org/10.1016/j.midw.2013.11.003

Hauck, Y., Fenwick, J., Downie, J. \& Butt, J. (2007). The influence of childbirth expectations on Western Australian women's perceptions of their birth experience. Midwifery, 23, 235-247. https://doi.org/10.1016/j.midw.2006.02.002

Hodnett, E. D. (2002). Pain and women's satisfaction with the experience of childbirth: A systematic review. American Journal of Obstetrics and Gynecology, 186, 160-172. https://doi.org/10. 1016/S0002-9378(02)70189-0

Hodnett, E. D., Gates, S., Hofmeyr, G. J. \& Sakala, C. (2012). Continuous support for women during childbirth. The Cochrane database of systematic reviews, 10, CD003766. https://doi.org/10. 1002/14651858.CD003766.pub4

Hodnett, E. D. \& Simmons-Tropea, D. A. (1987). The labour agentry scale: Psychometric properties of an instrument measuring control during childbirth. Research in Nursing \& Health, 10, 301 -310. https://doi.org/10.1002/nur.4770100503

Hu, L.-t. \& Bentler, P. M. (1999). Cutoff criteria for fit indexes in covariance structure analysis: Conventional criteria versus new alternatives. Structural Equation Modeling: A Multidisciplinary Journal, 6(1), 1 -55. https://doi.org/10.1080/1070551990 9540118

Larkin, P., Begley, C. M. \& Devane, D. (2009). Women's experiences of labour and birth: An evolutionary concept analysis. Midwifery, 25(2), e49-59. https://doi.org/10.1016/j.midw.2007.07.010 
Lavender, T., Walkinshaw, S. A. \& Walton, I. (1999). A prospective study of women's views of factors contributing to a positive birth experience. Midwifery, 15(1), 40-46. https://doi.org/10. 1016/S0266-6138(99)90036-0

Maimburg, R. D., Væth, M., Hvidman, L., Dürr, J. \& Olsen, J. (2013). Women's worries in first pregnancy: Results from a randomised controlled trial. Sexual \& Reproductive Healthcare, 4, 129-131. https://doi.org/10.1016/j.srhc.2013.10.001

McCrea, B. H. \& Wright, M. E. (1999). Satisfaction in childbirth and perceptions of personal control in pain relief during labour. Journal of advanced nursing, 29, 877-884. https://doi.org/10. 1046/j.1365-2648.1999.00961.x

Moosbrugger, H. \& Kelava, A. (2012). Testtheorie und Fragebogenkonstruktion (Springer-Lehrbuch, 2., aktual. und überarb. Aufl.). Berlin: Springer. https://doi.org/10.1007/978-3-642-20072-4

Mori, R., Tokumasu, H., Pledge, D. \& Kenyon, S. (2011). High dose versus low dose oxytocin for augmentation of delayed labour. The Cochrane database of systematic reviews, 10, CD007201. https://doi.org/10.1002/14651858.CD007201.pub2

Nystedt, A., Högberg, U. \& Lundman, B. (2005). The negative birth experience of prolonged labour: A case-referent study. Journal of clinical nursing, 14, 579-586. https://doi.org/10.1111/j. 1365-2702.2004.01105.x

Öhman, S. G., Grunewald, C. \& Waldenström, U. (2003). Women's worries during pregnancy: Testing the Cambridge Worry Scale on 200 Swedish women. Scandinavian Journal of Caring Sciences, 17, 148-152. https://doi.org/10.1046/j.1471-6712.2003. 00095.x

Pang, M. W., Leung, T. N., Lau, T. K. \& Hang Chung, T. K. (2008). Impact of first childbirth on changes in women's preference for mode of delivery: Follow-up of a longitudinal observational study. Birth, 35, $121-128$. https://doi.org/10.1111/j.1523-536X. 2008.00225.x

Pantlen, A. \& Rohde, A. (2001). Psychische Auswirkungen traumatisch erlebter Entbindungen. Zentralblatt für Gynäkologie, 123(1), 42 - 47. https://doi.org/10.1055/s-2001-12025

Peñacoba-Puente, C., Monge, F. J. C. \& Morales, D. M. (2011). Pregnancy worries: A longitudinal study of Spanish women. Acta Obstetricia et Gynecologica Scandinavica, 90, 1030 - 1035. https://doi.org/10.1111/j.1600-0412.2011.01208.x

Righetti-Veltema, M., Conne-Perréard, E., Bousquet, A. \& Manzano, J. (1998). Risk factors and predictive signs of postpartum depression. Journal of affective disorders, 49, 167-180. https:// doi.org/10.1016/S0165-0327(97)00110-9

Robinson, P. N., Salmon, P. \& Yentis, S. M. (1998). Maternal satisfaction. International Journal of Obstetric Anesthesia, 7(1), 32 - 37. https://doi.org/10.1016/S0959-289X(98)80026-5

Ryding, E. L. (1993). Investigation of 33 women who demanded a cesarean section for personal reasons. Acta Obstetricia et Gynecologica Scandinavica, 72, 280 -285. https://doi.org/10. 3109/00016349309068038

Salmon, P. \& Drew, N. C. (1992). Multidimensional assessment of women's experience of childbirth: Relationship to obstetric procedure, antenatal preparation and obstetric history. Journal of psychosomatic research, 36, 317-327. https://doi.org/10. 1016/0022-3999(92)90068-D

Salmon, P., Miller, R. \& Drew, N. C. (1990). Women's anticipation and experience of childbirth: The independence of fulfilment, unpleasantness and pain. British Journal of Medical Psychology, 63, 255-259. https://doi.org/10.1111/j.2044-8341.1990. tb01617.x

Schafer, J. L. \& Graham, J. W. (2002). Missing data: Our view of the state of the art. Psychological Methods, 7, 147-177. https:// doi.org/10.1037//1082-989X.7.2.147

Simkin, P. (1991). Just another day in a woman's life? Women's long-term perceptions of their first birth experience - Part I.
Birth, 18, 203 -210. https://doi.org/10.1111/j.1523-536X.1991. tb00103.x

Simkin, P. (1992). Just another day in a woman's life? Part 11: Nature and consistency of women's long-term memories of their first birth experiences. Birth, 19(2), 64-81. https://doi. org/10.1111/j.1523-536X.1992.tb00382.x

Slade, P., MacPherson, S. A., Hume, A. \& Maresh, M. (1993). Expectations, experiences and satisfaction with labour. British Journal of Clinical Psychology, 32, 469 - 483. https://doi.org/10. 1111/j.2044-8260.1993.tb01083.x

Soet, J. E., Brack, G. A. \& Dilorio, C. (2003). Prevalence and predictors of women's experience of psychological trauma during childbirth. Birth, 30(1), 36 - 46. https://doi.org/10.1046/j.1523536X.2003.00215.x

Stadlmayr, W., Bitzer, J., Hösli, I., Amsler, F., Leupold, J., Schwendke-Kliem, A. et al. (2001). Birth as a multidimensional experience: Comparison of the English- and German-language versions of Salmon's Item List. BJOG: Journal of Psychosomatic Obstetrics \& Gynecology, 22(4), 205-214. https://doi.org/10. 3109/01674820109049975

Waldenström, U., Borg, I. M., Olsson, B., Sköld, M. \& Wall, S. (1996). The childbirth experience: A study of 295 new mothers. Birth, 23, 144-153. https://doi.org/10.1111/j.1523-536X.1996.tb00475.x

Waldenström, U., Hildingsson, I. \& Ryding, E. L. (2006). Antenatal fear of childbirth and its association with subsequent caesarean section and experience of childbirth. BJOG: An International Journal of Obstetrics and Gynaecology, 113, 638-646. https://doi.org/10.1111/j.1471-0528.2006.00950.x

Waldenström, U. (1999). Experience of labor and birth in 1111 women. Journal of psychosomatic research, 47, 471-482. https://doi.org/10.1016/S0022-3999(99)00043-4

Waldenström, U., Hildingsson, I., Rubertsson, C. \& Radestad, I. (2004). A negative birth experience: Prevalence and risk factors in a national sample. Birth, 31(1), 17-27. https://doi.org/10. 1111/j.0730-7659.2004.0270.x

Walker, K. F., Wilson, P., Bugg, G. J., Dencker, A. \& Thornton, J. G. (2015). Childbirth experience questionnaire: Validating its use in the United Kingdom. BMC pregnancy and childbirth, 15(86), 1-8. https://doi.org/10.1186/s12884-015-0513-4

Watkins, M. W. (2017). The reliability of multidimensional neuropsychological measures: From alpha to omega. The Clinical neuropsychologist, 31, 1113-1126. https://doi.org/10.1080/ 13854046.2017 .1317364

Wijma, K., Söderquist, J. \& Wijma, B. (1997). Posttraumatic stress disorder after childbirth: A cross sectional study. Journal of anxiety disorders, 11, 587 - 597. https://doi.org/10.1016/S08876185(97)00041-8

Wiklund, I., Edman, G., Ryding, E.-L. \& Andolf, E. (2008). Expectation and experiences of childbirth in primiparae with caesarean section. BJOG: An InternationalJjournal of Obstetrics and Gynaecology, 115, 324-331. https://doi.org/10.1111/j.14710528.2007.01564.x

\section{Förderung}

Open Access-Veröffentlichung ermöglicht durch die ChristianAlbrechts-Universität

\section{Prof. Dr. Anya Pedersen}

Lehrstuhl für Klinische Psychologie und Psychotherapie Christian-Albrechts-Universität zu Kiel

Olshausenstraße 62

$24118 \mathrm{Kiel}$

pedersen@psychologie.uni-kiel.de 